\title{
A note on optimality conditions to interval optimization problems
}

\section{Y. Chalco-Cano ${ }^{1}$ R. Osuna-Gómez ${ }^{2}$ B. Hernández-Jiménez ${ }^{3}$ H. Román-Flores ${ }^{1}$}

\author{
${ }^{1}$ Instituto de Alta Investigación, Universidad de Tarapacá, Casilla 7D, Arica, Chile \\ ${ }^{2}$ Departamento de Estadística e I.O., Universidad de Sevilla, Spain \\ ${ }^{3}$ Dpto. de Econ., Mét. Cuant. e H. Económica. Área de Est. e I.O., Universidad Pablo de Olavide, Spain
}

\begin{abstract}
In this article we examine to necessary and sufficient optimality conditions for interval optimization problems. We introduce a new concept of stationary point for an interval-valued function based on the $g H$-derivative. We show the importance the this concept from a practical and computational point of view. We introduce a new concept of invexity for $g H$-differentiable interval-valued function which generalizes previous concepts and we prove that it is a sufficient optimality condition. Finally, we show that the concepts of differentiability, convexity and invexity for interval-valued functions based on the differentiability, convexity and invexity of its endpoint functions are not adequate tools for interval optimization problems.
\end{abstract}

Keywords: Interval optimization, sufficient conditions, generalized convexity

\section{Introduction}

The stationary point notion for interval optimization problems has been introduced in previous articles. With these notions we can obtain necessary optimality conditions only for some class of interval optimization problems $[15,16,19]$ or they are proved under restrictive conditions (for instance, comparable interval-valued functions)[11]. In these notions very restrictive concepts of differentiability for interval-valued functions were used. For instance, Hukuhara derivative or derivative based on the differentiability of the endpoint functions $[1,14,15,16,17,18,19]$.

Here we introduce a new notion of stationary point for interval-valued functions considering the generalized Hukuhara derivative, which is the most general concept so far.

Another main part in optimization theory is establishing sufficient optimality conditions. In the literature we can find articles devoted on this issue. In general the considered conditions are based on the convexity or generalized convexity of the endpoint functions of an interval-valued function $[1,9,14,15,16,17,18,19]$. We show that these conditions are very restrictive. We introduce a new concept of invexity for generalized Hukuhara differentiable interval-valued functions and show that invexity is such that each stationary point is minimum. In addition we show that invex intervalvalued functions are conditions more adequate than previous work.

\section{The space of intervals and interval-valued functions}

We denote by $\mathcal{K}_{C}$ the family of all bounded closed intervals in $\mathbb{R}$, i.e.,

$$
\mathcal{K}_{C}=\{[\underline{a}, \bar{a}] / \underline{a}, \bar{a} \in \mathbb{R} \text { and } \underline{a} \leq \bar{a}\},
$$

Given $A=[\underline{a}, \bar{a}] \in \mathcal{K}_{C}, \underline{a}$ and $\bar{a}$ stand for the lower and upper bounds of $A$, respectively. For $A=$ $[\underline{a}, \bar{a}], B=[\underline{b}, \bar{b}] \in \mathcal{K}_{C}$ and $\lambda \in \mathbb{R}$ we consider the following operations

$$
\begin{gathered}
A+B=[\underline{a}, \bar{a}]+[\underline{b}, \bar{b}]=[\underline{a}+\underline{b}, \bar{a}+\bar{b}], \\
\lambda \cdot A=\lambda[\underline{a}, \bar{a}]=\left\{\begin{array}{lll}
{[\lambda \underline{a}, \lambda \bar{a}]} & \text { if } \quad \lambda \geq 0, \\
{[\lambda \bar{a}, \lambda \underline{a}]} & \text { if } \quad \lambda<0 .
\end{array}\right.
\end{gathered}
$$

From (1) and (2) we have $-A=[-\bar{a},-\underline{a}]$ and $B-$ $A=B+(-A)=[\underline{b}-\bar{a}, \bar{b}-\underline{a}]$. The space $\mathcal{K}_{C}$ with operations (1) and (2) is not a linear space since an interval does not have inverse element and therefore subtraction does not have adequate properties (see $[3,13])$.

More recently, Stefanini and Bede in [13] have introduced the following difference between two intervals.

Definition 1 ([13]) The generalized Hukuhara difference of two intervals, $A$ and $B,(g H$-difference) is defined as follows

$$
A \ominus_{g H} B=C \Leftrightarrow\left\{\begin{array}{l}
(a) A=B+C, \\
(b) B=A+(-1) C .
\end{array}\right.
$$

This difference has many interesting new properties, for example $A \ominus_{g} A=\{0\}=[0,0]$. Also, the $g H$-difference of two intervals $A=[\underline{a}, \bar{a}]$ and $B=[\underline{b}, \bar{b}]$ always exists and it is equal to (see Proposition 4 in [13])

$A \ominus_{g H} B=[\min \{\underline{a}-\underline{b}, \bar{a}-\bar{b}\}, \max \{\underline{a}-\underline{b}, \bar{a}-\bar{b}\}]$.

Give two intervals $A=[\underline{a}, \bar{a}]$ and $B=[\underline{b}, \bar{b}]$ we have

$$
\begin{gathered}
A \leqq B \Longleftrightarrow \underline{a} \leq \underline{b} \text { and } \bar{a} \leq \bar{b} ; \\
A \preceq B \Longleftrightarrow A \leqq B \text { and } A \neq B ; \\
A \prec B \Longleftrightarrow \underline{a}<\underline{b} \text { and } \bar{a}<\bar{b} .
\end{gathered}
$$


The order relation $\leqq$ was initially introduced in [10] and used in interval optimization problems, see $[4$, $7,8,15,16]$.

\section{1. $g H$-differentiable interval-valued functions}

In this paper $T$ denotes an open set in $\mathbb{R}$ and a function $F: T \rightarrow \mathcal{K}_{C}$ will be called interval-valued function. For each $x$ in the domain we denote $F(x)=[f(x), \bar{f}(x)]$ such that $\underline{f}(x) \leq \bar{f}(x)$. The functions $f$ and $\bar{f}$ will be called, respectively, the lower and the upper (endpoint) functions of $F$.

Based on the $g H$-difference, the following definition of differentiability for interval-valued functions was introduced in Stefanini and Bede in [13].

Definition 2 ([13]) The $g H$-derivative of an interval-valued function $F: T \rightarrow \mathcal{K}_{C}$ at $x_{0} \in T$ is defined as

$$
F^{\prime}\left(x_{0}\right)=\lim _{h \rightarrow 0} \frac{F\left(x_{0}+h\right) \ominus_{g H} F\left(x_{0}\right)}{h} .
$$

If $F^{\prime}\left(x_{0}\right) \in \mathcal{K}_{C}$ satisfying (3) exists, we say that $F$ is generalized Hukuhara differentiable ( $g H$ differentiable) at $x_{0}$. We say that $F$ is $g H$ differentiable on $T$ if $F$ is $g H$-differentiable at each point $x_{0} \in T$.

In (3) the limits are taken in the metric space $\left(\mathcal{K}_{C}, H\right)$, where $H$ is defined by

$$
H(A, B)=\max \{|\underline{a}-\underline{b}|,|\bar{a}-\bar{b}|\} .
$$

Next we give the following result which connects $g H$-differentiability of $F$ and the differentiability of its endpoint functions $\underline{f}$ and $\bar{f}$.

Theorem 1 ([3]) Let $F: T \rightarrow \mathcal{K}_{C}$ be a function. Then, $F$ is $g H$-differentiable at $x_{0} \in T$ if and only if one of the following cases hold:

(a) $\underline{f}$ and $\bar{f}$ are differentiable at $x_{0}$ and

$F^{\prime}\left(x_{0}\right)=\left[\min \left\{\underline{f}^{\prime}\left(x_{0}\right), \bar{f}^{\prime}\left(x_{0}\right)\right\}, \max \left\{\underline{f}^{\prime}\left(x_{0}\right), \bar{f}^{\prime}\left(x_{0}\right)\right\}\right] ;$

(b) the lateral derivatives $\underline{f}_{-}^{\prime}\left(x_{0}\right), \underline{f}_{+}^{\prime}\left(x_{0}\right), \bar{f}_{-}^{\prime}\left(x_{0}\right)$ and $\bar{f}_{+}^{\prime}\left(x_{0}\right)$ exist and satisfy $\underline{f}_{-}^{\prime}\left(x_{0}\right)=\bar{f}_{+}^{\prime}\left(x_{0}\right)$ and $\underline{f}_{+}^{\prime}\left(x_{0}\right)=\bar{f}_{-}^{\prime}\left(x_{0}\right)$. Moreover

$$
\begin{aligned}
& F^{\prime}\left(t_{0}\right) \\
= & {\left[\min \left\{\underline{f}_{-}^{\prime}\left(x_{0}\right), \bar{f}_{-}^{\prime}\left(x_{0}\right)\right\}, \max \left\{\underline{f}_{-}^{\prime}\left(x_{0}\right), \bar{f}_{-}^{\prime}\left(x_{0}\right)\right\}\right] } \\
= & {\left[\min \left\{\underline{f}_{+}^{\prime}\left(x_{0}\right), \bar{f}_{+}^{\prime}\left(x_{0}\right)\right\}, \max \left\{\underline{f}_{+}^{\prime}\left(x_{0}\right), \bar{f}_{+}^{\prime}\left(x_{0}\right)\right\}\right] }
\end{aligned}
$$

There are other concepts of derivative for intervalvalued functions which were used in interval optimization: Hukuhara derivative ( $H$-derivative $[6])$ and endpoint derivative (e-derivative). It well known that if $F$ is $H$-differentiable then it possesses the property that the diameter $\operatorname{len}(F(t)$ ) (lenght of $F(t))$ is nondecreasing as $t$ increases $[2,3,13]$. Thus
$H$-derivative is a concept very restrictive. On the other hand, the $e$ derivative is based on the derivative of its endpoint functions of $F$.

Definition 3 Let $F: T \rightarrow \mathcal{K}_{C}$ be an intervalvalued function. We say that $F$ is endpoint differentiable (e-differentiable for short) if and only if the endpoint functions $\underline{f}$ and $\bar{f}$ are differentiable.

We note that initially $e$-differentiability was introduced as weakly differentiability [15].

We can clearly see that the $g H$-derivative is a more general concept than the Hukuhara and endpoint derivatives. On the other hand, both Hukuhara and endpoint derivative are very restrictive as we show in the following example.

Example 1 ([4]) We consider the interval-valued function $F: \mathbb{R} \rightarrow \mathcal{K}_{C}$ defined by $F(x)=A \cdot x$, where $A=[1,2]$. Clearly we can see that $F$ is an extension of a linear function to interval-valued context. From (2) we have

$$
F(x)=\left\{\begin{array}{lll}
{[x, 2 x]} & \text { if } \quad x \geq 0 \\
{[2 x, x]} & \text { if } \quad x<0
\end{array}\right.
$$

We can see that the endpoint functions $f$ and $\bar{f}$ are not differentiable at $t=0$ and so $F$ is not $H$ differentiable and it is not e-differentiable. However $F$ is $g H$-differentiable on $\mathbb{R}$ and $F^{\prime}(x)=A$ for all $x \in \mathbb{R}$.

\section{Interval optimization problems}

Since the value $F(x)$ is a closed interval, we need to interpret the minimization meaning. The most straightforward way is to invoke the order relations between intervals. However, the order relation is not a total order in $\mathcal{K}_{C}$. So, we follow a similar solution concept as that used in multiobjective programming problem to to define a minimum.

In $[1,4,5,9,14,15,16,17,18,19]$ the authors considered the following concept of minimum for interval-valued functions.

Definition 4 Let $F: T \rightarrow \mathcal{K}_{C}$ be an intervalvalued function.

(i) $x^{*} \in T$ is said to be a (local) minimum if there does not exist $x \in T\left(\exists \delta>0, x \in B\left(x^{*}, \delta\right) \cap S\right)$ such that $F(x) \preceq F\left(x^{*}\right)$;

(ii) $x^{*} \in T$ is said to be a (local) weak minimum if there does not exist $x \in T\left(\exists \delta>0, x \in B\left(x^{*}, \delta\right) \cap S\right)$ such that $F(x) \prec F\left(x^{*}\right)$.

Clearly, if $x^{*} \in T$ is minimum then it is weak minimum.

\subsection{Necessary conditions}

Next we propose a new definition of stationary point for interval-valued functions. 
Definition 5 Let $F: T \rightarrow \mathcal{K}_{C}$ a $g H$-differentiable interval-valued function and $x^{*} \in T$. We say that $x^{*}$ is a stationary point for $F$ if $0 \in F^{\prime}\left(x^{*}\right)$.

Now is turn to present a new necessary optimality condition.

Theorem 2 Let $F$ be a $g H$-differentiable intervalvalued function. If $x^{*}$ is a local weak minimum of $F$ then $x^{*}$ is a stationary point for $F$.

Proof Arguing by contradiction let us suppose that $0 \notin F^{\prime}\left(x^{*}\right)$. Then, two cases are possible:

$$
\text { (i) } F^{\prime}\left(x^{*}\right) \subset \mathbb{R}^{+} \text {or }(\text { ii }) F^{\prime}\left(x^{*}\right) \subset \mathbb{R}^{-} \text {. }
$$

We suppose (i) and let us consider each one of the two different forms (a) and (b) to write $F^{\prime}\left(x^{*}\right)$ in Theorem 1.

(ia) If $F^{\prime}\left(x^{*}\right) \subset \mathbb{R}^{+}$then $\underline{f}^{\prime}\left(x^{*}\right), \bar{f}^{\prime}\left(x^{*}\right)>0$ and so $f$ and $\bar{f}$ are increasing functions in a neighborhood of $x^{*}$. Considering $\epsilon$ small enough, we get

$$
\underline{f}\left(x^{*}-\epsilon\right)<\underline{f}\left(x^{*}\right) \text { and } \bar{f}\left(x^{*}-\epsilon\right)<\bar{f}\left(x^{*}\right) .
$$

So $F\left(x^{*}-\epsilon\right) \prec F\left(x^{*}\right)$.

(ib) $F^{\prime}\left(x^{*}\right) \subset \mathbb{R}^{+}$implies that $\bar{f}_{-}^{\prime}\left(x^{*}\right)$ and $\underline{f}_{-}^{\prime}\left(x^{*}\right)>$ 0 , then

$$
\bar{f}_{-}^{\prime}\left(x^{*}\right)=\lim _{h \rightarrow 0^{-}} \frac{\bar{f}\left(x^{*}+h\right)-\bar{f}\left(x^{*}\right)}{h}>0 .
$$

Since $h<0$, there exists $\epsilon_{1}>0$ such that $\forall h \in$ $\left(-\epsilon_{1}, 0\right)$,

$$
\bar{f}\left(x^{*}+h\right)<\bar{f}\left(x^{*}\right) .
$$

In the same form, there exists $\epsilon_{2}$ such that $\forall h \in$ $\left(-\epsilon_{2}, 0\right)$,

$$
\underline{f}\left(x^{*}+h\right)<\underline{f}\left(x^{*}\right) .
$$

Considering $h<0$ such that $|h|<\min \left\{\epsilon_{1}, \epsilon_{2}, \delta\right\}$, then

$$
F\left(x^{*}+h\right) \prec F\left(x^{*}\right) .
$$

Arguing in the same way than case $(i a)$ and $(i b)$, for case $(i i a)$ and $(i i b)$ we get also a contradiction with $x^{*}$ is a minimum.

Corollary 1 Let $F$ be a $g H$-differentiable intervalvalued function. If $x^{*}$ is a local minimum of $F$ then $x^{*}$ is a stationary point for $F$.

We must emphasize the importance of Theorem 2 since this allows to select potential candidates to be minimum for an interval-valued function just by checking whether 0 belongs to a closed and bounded interval. On the other hand, we used $g H$-derivative to obtain this result. It generalize previous articles where the authors used $H$-derivative and $e$ derivative. The following result show this fact.

Example 2 We consider the interval-valued function $F: \mathbb{R} \rightarrow \mathcal{K}_{C}$ defined by $F(x)=[-1,1] \cdot x$. From (2) we have

$$
F(x)=[-|x|,|x|] .
$$

In this case $F$ is $g H$-differentiable and $F^{\prime}(x)=$ $[-1,1]$ for all $x \in \mathbb{R}$. Clearly any real number is a stationary point for $F$ and so these are also candidates to be minimum for $F$. In fact, all real number are minimum for $F$.

From Example 2 we can see that the endpoint functions $f$ and $\bar{f}$ are not differentiable and so $F$ is not $H$-differentiable and it is not $e$-differentiable. Therefore concepts of stationary point for $F$ using $H$-derivative and $e$-derivative are more restrictive.

\subsection{Sufficient conditions}

Of course the converse of Theorem 2 is not valid. For instance, if we consider $F(x)=[1,2] \cdot x^{3}$, we have that $x^{*}=0$ is a stationary point for $F$ but it is not a minimum.

In this subsection we study sufficient conditions for a stationary point to be a minimum of $F$.

The following definition of convexity for intervalvalued functions is well-known in the literature, for instance see $[4,5,15,16,17]$ and references therein.

Definition 6 An interval-valued function $F: T \rightarrow$ $\mathcal{K}_{C}$ is said to be convex on a convex set $T$ if and only if for any $x, y \in T$ and $\lambda \in[0,1]$

$$
F(\lambda x+(1-\lambda) y) \leqq \lambda F(x)+(1-\lambda) F(y) .
$$

If (4) satisfies for $\prec$ then we say that $F$ is strict convex.

We want to note that the convexity is equivalent to convexity of the endpoint functions.

Proposition 1 ([4,15,16, 18]) $F$ is convex if and only if $f$ and $\bar{f}$ are convex.

From interval arithmetic we can see that the concept of convexity is very restrictive such as we show in the following example.

Example 3 We consider the interval-valued function $F: \mathbb{R} \rightarrow \mathcal{K}_{C}$ defined by $F(x)=A \cdot x$, with $A=[\underline{a}, \bar{a}] \subset \mathbb{R}^{+}$, which is a extension of a linear function to interval-valued context. Then from interval arithmetic (2) we have

$$
F(x)=\left\{\begin{array}{lll}
{[\underline{a} x, \bar{a} x]} & \text { if } \quad x \geq 0 \\
{[\bar{a} x, \underline{a} x]} & \text { if } \quad x<0 .
\end{array}\right.
$$

Since $\underline{f}$ is not convex then $F$ is not convex.

We have the following necessary condition for convex interval-valued functions.

Theorem 3 ([5]) Let $F: T \rightarrow \mathcal{K}_{C}$ be a $g H$ differentiable interval-valued function. If $F$ is convex then

$$
F(x) \ominus_{g H} F(y) \geqq F^{\prime}(y) \cdot(x-y),
$$

for all $x, y \in T$. 
The converse of the Theorem 3 is not valid. For instance, if we consider $F$ as in the Example 3 we have that $F$ is not convex but it satisfies the condition (5).

Based on the previous theorem we introduced the following concept.

Definition 7 Let $F: T \rightarrow \mathcal{K}_{C}$ be a $g H$ differentiable interval-valued function and let $T$ be an invex set. Then $F$ is said to be invex on $K$ if for all $x, y \in T$, there exists $\eta: T \times T \rightarrow \mathbb{R}$ such that

$$
F(x) \ominus_{g H} F(y) \geqq F^{\prime}(y) \cdot \eta(x, y),
$$

for all $x, y \in T$.

If (6) satisfies to $\succ$ then we say that $F$ is strict invex.

Clearly if $F$ is convex then it is invex and the converse is not valid. Also we have the following consequence.

Corollary 2 Let $F: T \rightarrow \mathcal{K}_{C}$ be a interval-valued function and let $T$ be an invex set. If for all $x, y \in$ $T$, there exists $\eta: T \times T \rightarrow \mathbb{R}$ such that

$$
F(x) \geqq F^{\prime}(y) \cdot \eta(x, y)+F(y),
$$

for all $x, y \in T$. Then $F$ is invex.

Note that the converse in Corollary 2 is not valid. In addition, we note that an interval-valued function that satisfies the condition (7) was called invex in the article [12]. So, the definition given by (6) is more general.

Next we give conditions such that a stationary point is a minimum point based on convexity and invexity.

Theorem 4 Let $F: T \rightarrow \mathcal{K}_{C}$ be a interval-valued function. If $F$ is invex then each stationary point for $F$ is a weak minimum point for $F$.

Proof We suppose that $x^{*} \in T$ is a stationary point for $F$ and it is not a weak minimum for $F$, then there exists another $x \in T$, with $x \neq x^{*}$, such that $F(x) \prec F\left(x^{*}\right)$. Now, since $F$ is invex there exists $\eta: K \times K \rightarrow \mathbb{R}$ such that

$$
F(x) \ominus_{g H} F\left(x^{*}\right) \geqq F^{\prime}\left(x^{*}\right) \cdot \eta(x, y) .
$$

Taking on account that $F(x) \prec F\left(x^{*}\right)$ and Proposition 2 part (v) in [5] we obtain

$$
F\left(x^{*}\right) \ominus_{g H} F\left(x^{*}\right) \succ F^{\prime}\left(x^{*}\right) \cdot \eta(x, y),
$$

equivalently

$$
\{0\} \succ F^{\prime}\left(x^{*}\right) \cdot \eta(x, y) .
$$

Thus $\{0\} \succ F^{\prime}\left(x^{*}\right)$ or $\{0\} \prec F^{\prime}\left(x^{*}\right)$. Which contradicts the hypothesis. $\square$

Theorem 5 Let $F: T \rightarrow \mathcal{K}_{C}$ be a interval-valued function. If $F$ is strict invex then each stationary point for $F$ is a minimum point for $F$.
Proof It follow on the same argument of the demonstration of the Theorem $4 . \square$

In the literature we can find articles where the authors considered other concepts of invexity and generalized invexity for $F$ based on the invexity and generalized invexity of its endpoint function as a generalization of convexity (based in the Proposition 1).

Definition $8[1,14,18]$ Let $F: T \rightarrow \mathcal{K}_{C}$ be an $e$ differentiable interval-valued function and let $T$ be an invex set. Then $F$ is said to be endpoint invex (e-invex, for short) respect to $\eta$ if and only if $\underline{f}$ and $\bar{f}$ are invex with respect of same $\eta$.

The $e$-invexity is a concept very restrictive in two way: first by the use of $e$-derivative and second by claim the invexity of the endpoint functions which is restrictive such as occur with the convexity. In fact if consider $F$ defined as in the Example 3 we have that $F$ is not invex since $f$ is not invex but it is invex since it satisfies the condition (6) with $\eta(x, y)=x-y$.

The following example show the advantages of the concept of invexity given here.

Example 42 We consider the interval-valued function $F: \mathbb{R} \rightarrow \mathcal{K}_{C}$ defined as in the Exampled, i.e. $F(x)=[-1,1] \cdot x$, equivalently

$$
F(x)=[-|x|,|x|] .
$$

We have that $F$ is $g H$-differentiable and $F^{\prime}(x)=$ $[-1,1]$ for all $x \in \mathbb{R}$. Clearly any real number is a stationary point for $F$ and so these are also candidates to be weak minimum for $F$.

On the other hand, $F$ is an invex interval-valued funtion respecto to $\eta(x, y)=|x|-|y|$ for all $x, y \in \mathbb{R}$. Therefore, from Theorem 4, any real number is a weak minimum point.

Note that in this case $F$ is not e-differentiable and $F$ is not e-invex.

\section{Conclusions}

We have introduced new concept of stationary point for an interval-valued function based on the $g H$ derivative. We must emphasize the importance that this concept has from a practical and computational point of view. We have introduced a new concept of invexity which generalizes previous approaches and we have shown that it is a sufficient optimality condition.

On the other hand, we have shown that the concepts of $e$-differentiability, convexity and $e$-invexity are not adequate tools for interval optimization problems.

\section{Acknowledgments.}

This paper has been supported by Fondecyt-Chile through projects 1120665, 1151154 and 1151159, 
and Ministerio de Ciencia e Innovación, Spain, through grant MTM2010-15383.

\section{References}

[1] I. Ahmad, A. Jayswal and J. Banerjee, On interval-valued optimization problems with generalized invex functions, Journal of Inequalities and Applications, 2013:313, 2013.

[2] Y. Chalco-Cano, H. Román-Flores and M.D. Jiménez-Gamero, Generalized derivative and $\pi$ derivative for set-valued functions, Information Sciences, 181: 2177-2188, 2011.

[3] Y. Chalco-Cano, A. Rufián-Lizana, H. RománFlores, M.D. Jiménez-Gamero, Calculus for interval-valued functions using generalized Hukuhara derivative and applications, Fuzzy Sets and Systems, 219: 49-67, 2013.

[4] Y. Chalco-Cano, W.A. Lodwick and RufiánLizana, Optimality conditions of type KKT for optimization problem with interval-valued objective function via generalized derivative,Fuzzy Optimization and Decision Making, 12: 305-322 2013.

[5] Y. Chalco-Cano, Generalized Hukuhara differentiability and interval optimization problems, Proceding of Third Congress of Fuzzy Systems, João Pessoa, August 2014.

[6] M. Hukuhara, Integration des applications mesurables dont la valeur est un compact convexe. Funkcialaj Ekvacioj, 10: 205-223, 1967.

[7] M. Inuiguchi and Y. Kume, Goal programming problems with interval coefficients and target intervals. European Journal of Operational Research, 52: 345-360, 1991.

[8] H. Ishibushi and H. Tanaka, Multiobjective programming in optimization of the interval objective function. European Journal of Operational Research, 48: 219-225, 1990.

[9] A. Jayswal, Ioan Stancu-Minasian, I. Ahmad, On sufficiency and duality for a class of intervalvalued programming problems, Applied Mathematics and Computation, 218: 4119-4127, 2011.

[10] U. W. Kulisch and W. L. Miranker, Computer arithmetic in theory and in practice, 1nd edition, Academic Pr., 1981.

[11] Pirzada U.M., Pathak V.D., Newton method for solving the multi-variable fuzzy optimization problem, Journal Optimization Theory and Applications, 156: 867-881, 2013.

[12] A. Rufián-Lizana, Y. Chalco-Cano, R. OsunaGómez, G. Ruiz-Garzón, On invex fuzzy mappings and fuzzy variational-like inequalities, Fuzzy Sets and Systems, 200: 84-98, 2012.

[13] L. Stefanini and B. Bede, Generalized Hukuhara differentiability of interval-valued functions and interval differential equations, Nonlinear Analysis, 71: 1311-1328, 2009.

[14] Y. Sun, L. Wang, Optimality conditions and duality in nondifferentiable interval valued pro- gramming, Journal Management Optimization, 9: 131-142, 2013.

[15] H-C. Wu, The Karush-Kuhn-Tucker optimality conditions in an optimization problem with interval-valued objective function. European Journal of Operational Research, 176: 4659, 2007.

[16] H-C. Wu, The Karush-Kuhn-Tucker optimality conditions in multiobjective programming problems with interval-valued objective functions. European Journal of Operational Research, 196: 49-60, 2009.

[17] H-C. Wu, Duality theory for optimization problems with interval-valued objective functions, J. Optim Theory Appl., 144: 615-628, 2009.

[18] J. Zhang, S. Liu, L. Li, Q. Feng, The KKT optimality conditions in a class of generalized convex optimization problems with an intervalvalued objective function, Optimization Letters, 8: 607-631, 2014.

[19] Jianke Zhang, Optimality Condition and Wolfe Duality for Invex Interval-Valued Nonlinear Programming Problems, Journal of Applied Mathematics, 2013: 1-11, 2013. 